\title{
Apolipoprotein E Genotype Predicts 24-Month Bayley Scales Infant Development Score
}

\author{
ROBERT O. WRIGHT, HOWARD HU, EDWIN K. SILVERMAN, SHIRNG W. TSAIH, \\ JOEL SCHWARTZ, DAVID BELLINGER, EDUARDO PALAZUELOS, SCOTT T. WEISS, AND \\ MAURICIO HERNANDEZ-AVILA \\ Departments of Pediatrics [R.O.W.] and Neurology [D.B.], Children's Hospital Boston, Boston, \\ Massachusetts 02115, U.S.A.; The Channing Laboratory [R.O.W., H.H., E.K.S., S.W.T., J.S., S.T.W.], \\ Brigham and Women's Hospital, Harvard Medical School, Boston, Massachusetts 02115, U.S.A.; \\ Department of Environmental Health [R.O.W., H.H., S.W.T., J.S.], Harvard School of Public Health, \\ Boston, Massachusetts 02115, U.S.A.; American British Cowdray Hospital [E.P.], 18901 México City, \\ México; and Instituto Nacional de Salud Pública [E.P., M.H.-A.], Instituto Nacional de Perinatologia, \\ 62508 Mexico City, Centro de Investigacion en Salud, Poblacional, National Institute of Public Health \\ Mexico, 62508 Cuernavaca, México
}

\begin{abstract}
ABST
Apolipoprotein E (APOE) regulates cholesterol and fatty acid
metabolism, and may mediate synaptogenesis during neurode-
velopment. To our knowledge, the effects of APOE4 isoforms on
infant development have not been studied. This study was nested
within a cohort of mother-infant pairs living in and around
Mexico City. A multiple linear regression model was constructed
using the $24-m o$ Mental Development Index (MDI) of the Bayley
Scale as the primary outcome and infant APOE genotype as the
primary risk factor of interest. Regression models stratified on
APOE genotype were constructed to explore effect modification.
Of 311 subjects, 53 (17\%) carried at least one copy of the
APOE4 allele. Mean (SD) MDI scores among carriers with at
least one copy of APOE4 were 94.1 (14.3) and among E3/E2
carriers were 91.2 (14.0). After adjustment for covariates,
APOE4 carrier status was associated with a 4.4 point (95\%
\end{abstract}
Apolipoprotein E (ApoE, protein; APOE, gene) is an intracellular cholesterol and fatty acid transport protein that plays an important role in neuronal metabolism. The gene for this protein has been localized on chromosome 19, and there are three common alleles_E2, E3, and E4-which differ only on the basis of one or two amino acids on positions 112 and 158 $(1,2)$. ApoE is unique among lipoproteins in that it is involved in the recovery response of injured nerve tissue (3). In the brain, ApoE is synthesized by astrocytes and secreted into the extracellular space, where it then binds to cholesterol. There it

Received November 8, 2002; accepted May 26, 2003.

Correspondence: Robert O. Wright, MD, MPH, Division of Emergency Medicine, Children's Hospital, Boston, 300 Longwood Avenue, Boston, MA 02115, U.S.A.; email: robert.wright@channing.harvard.edu

Supported in part by grants from the National Institute of Environmental Health Sciences: K23 ES 00381 and R01 ES 007821

DOI: 10.1203/01.PDR.0000090927.53818.DE confidence interval: $0.1-8.7 ; p=0.04$ ) higher 24-mo MDI. In the stratified regression models, the negative effects for umbilical cord blood lead level on 24-mo MDI score was approximately 4-fold greater among APOE3/APOE2 carriers than among APOE4 carriers. These results suggest that subjects with the E4 isoform of APOE may have advantages over those with the E2 or E3 isoforms with respect to early life neuronal/brain development. (Pediatr Res 54: 819-825, 2003)

\author{
Abbreviations \\ APOE, apolipoprotein E gene \\ ApoE, apolipoprotein E protein \\ MDI, Mental Development Index \\ BSID, Bayley Scales of Infant Development
}

is taken up by neurons via various ApoE receptors and incorporated into cell membrane structures and myelin $(4,5)$. The metabolism of cholesterol is believed to play a major role in neurite outgrowth and synaptogenesis (1-3), processes that are critical to neurodevelopment. Although the APOE4 isoform has been demonstrated to be an important risk factor for neurodegeneration, the role of ApoE genetic variants in neurodevelopment is also becoming increasingly apparent (6-8).

In conjunction with our understanding of the role of cholesterol and ApoE in neurodevelopment is an increasing recognition that genetic factors that alter the host response to environmental toxins may be particularly important during critical developmental windows that occur during fetal life $(9,10)$. Because cholesterol and fatty acids are critical to brain development, genetic factors that regulate their metabolism may influence development independently or may serve as modifiers of the response to maternal diet or maternal exposure to 
neurotoxins. Genetic susceptibility may account for a large proportion of the variance in response to environmental toxins, such as lead. Because ApoE is critical to synaptogenesis processes involved in brain development, this raises the question of whether genetic variants of APOE may also be associated with differential effects on neurodevelopment. Given this background, we studied the role of APOE genotypes on infant development and explored interactions between APOE genotypes and fetal exposure to the neurotoxin lead.

\section{METHODS}

Study population. The study protocol was approved by the Human Subjects Committees of the National Institute of Public Health of Mexico, the participating hospitals, the Brigham and Women's Hospital, and the Harvard School of Public Health and was conducted under an established interinstitutional collaboration among Harvard University, the Center for Population Health Research of the National Institute of Public Health in Mexico, the American British Cowdray Medical Center, and the National Institute of Perinatology of Mexico. The study cohort was recruited from three maternity hospitals in Mexico City (Mexican Social Security Institute, Manuel Gea Gonzalez Hospital, and National Institute of Perinatology) and was designed to determine the effects of calcium supplementation during lactation on infant lead kinetics and neurodevelopment. We have previously reported the effect of maternal bone lead in predicting infant development in this cohort (11).

Between 1994 and 1995, 1382 women were found to be eligible for the trial. Exclusion criteria included no intention to breast-feed; factors that would interfere with data collection, such as living outside Mexico City; and conditions related to calcium metabolism and requirements, such as multiple fetuses, preeclampsia, or pregnancy-related hypertensive disorders or cardiac disease, gestational diabetes, history of repeated urinary infections, history of kidney stone formation, seizure disorder requiring daily medications, and ingestion of corticosteroids. Also excluded from the study were premature neonates $(<37 \mathrm{wk})$ and newborns with birth weight $<2000 \mathrm{~g}$. Baseline information on health status and social and demographic characteristics as well as written consent were obtained from all 1382 eligible participating mothers. One month postpartum, potential participants attended our research center. Of the 1382 initially eligible mother-infant pairs, 617 (44.6\%) agreed to participate in the trial. For the purposes of this analysis, 424 infants had archived blood available for genotyping. This study was conducted in these 424 infants.

Anthropometric data from the mother and the newborn and umbilical cord and maternal venous blood samples were gathered within $12 \mathrm{~h}$ of delivery. Information on estimated gestational age, based on the date of last menstrual period, and characteristics of the birth and newborn period were extracted from the medical records.

One month after delivery, consenting mother-infant pairs were invited to the research center for an evaluation that included measurement of maternal and infant lead biomarkers. Participating mother-infant pairs were subsequently assessed and interviewed when the infants were 24 mo of age using the
Bayley Scales of Infant Development-II (BSID-II; Spanish version). All participating mothers received a detailed explanation of the study and its procedures, as well as counseling on how to reduce environmental lead exposure.

Blood lead measurements. Umbilical cord blood samples were collected in trace metal-free tubes at delivery. Blood samples were analyzed using an atomic absorption spectrometry instrument (Perkin-Elmer 3000, Chelmsford, MA, USA) at the metals laboratory of the American British Cowdray Hospital in Mexico City. External blinded quality-control samples were provided throughout the study period by the Maternal and Child Health Bureau and the Wisconsin State Laboratory of Hygiene Cooperative Blood Lead Proficiency Testing Program. These analyses demonstrated acceptable precision and accuracy with a correlation coefficient of 0.99 and a mean difference of $0.17 \mu \mathrm{g} / \mathrm{dL}(\mathrm{SD}=0.68)$. The detection limit for this assay is $1 \mu \mathrm{g} / \mathrm{dL}$.

Measurements of child development and potential confounders. The BSID-II is a revision and restandardization of the BSID, one of the most widely used tests of infant development. Scores have been shown to be sensitive to a variety of prenatal, perinatal, and postnatal insults, including lead exposure (11-14). A Spanish version of the BSID (BSID-IIS) was developed by our research group before this study. The team administering the BSID-IIS was led and trained by Dr. Bellinger, with standardization and quality control checks conducted through reviews of videotaped interviews. Mental Development Index (MDI) scores at 24 mo of age were used as the primary outcome measure.

Maternal IQ, an important potential confounder, was assessed using the Vocabulary and Block Design components of the Wechsler Adult Intelligence Score, which has been translated into Spanish and used in Mexico. Of the 424 subjects with archived blood, 311 returned for a 24-mo BSID assessment.

Genotyping. Although each subject had signed an approved consent form signifying willingness to have blood archived for unspecified future testing, we took additional steps to protect the confidentiality of the APOE genotype data. To protect the subjects from any undue consequences that the results might have with respect to employment or health coverage, we created a new data file for the cohort, anonymized and kept separate from the master data file. Data on all exposure variables and covariates of interest were downloaded into this file, as were the APOE genotype data. All subject identifiers were then deleted from the new anonymous file, and the APOE genotype data were deleted from the master file. The genotyping and anonymization procedures were approved by the $\mathrm{Hu}-$ man Subjects Committee of Brigham and Women's Hospital.

Genotyping was performed on archived frozen samples of whole umbilical cord blood. High-molecular-weight DNA was extracted from white blood cells with commercially available PureGene Kits (Gentra Systems, Minneapolis, MN, USA). Extraction yielded an average of $20-40 \mu \mathrm{g}$ of DNA per ml of whole blood, depending on the white blood cell count. OD was measured using the 260/280 ratio $>1.7$ as an indication that the extraction was satisfactory. Before use, every sample underwent additional quality control by subjecting it to PCR ampli- 
fication (using a primer set amplifying the ACE D/I polymorphism for this purpose). After DNA quantification, samples were adjusted to TE buffer, partitioned into aliquots, and stored at $-80^{\prime} 45 \mathrm{C}$. For APOE genotyping, we used single-base extension with fluorescent dideoxynucleoside triphosphates (ddNTPs), which was been implemented in the ABI Prism SnaPshot ddNTP Primer Extension Kit (Applied Biosystems, Foster City, CA, USA). For the polymorphisms in APOE (E2, E3, E4), PCR primers were designed to produce amplicons of $500 \mathrm{bp}$, and PCR conditions were optimized. Genomic DNA was amplified using Amplitaq Gold (Perkin Elmer, Wellesley, MA) in a Tetrad PCR machine (MJ Research, Watertown, MA, USA). Residual PCR primers and unincorporated dNTPs were removed by digestion with shrimp alkaline phosphatase (Boehringer Mannheim, Indianapolis, IN) and E. coli exonuclease I (Amersham Pharmacia, Uppsala, Sweden). After heat inactivation of the shrimp alkaline phosphatase and exonuclease I, an unlabeled SNaPshot PCR primer (which contains sequence immediately $5^{\prime}$ to the SNP site) was added to the amplified DNA, along with ddNTPs labeled with distinct fluorescent tags (from Applied Biosystems). PCR with singlebase extension by Amplitaq DNA polymerase FS was performed, and incorporation of fluorescent ddNTPs was assessed by capillary electrophoresis on an ABI 3100 . The ABI 3100 output was analyzed with ABI Prism Genotyper Analysis Software, and genotype assignment was performed by comparison of SNaPshot results to DNA samples from laboratory and CEPH control genotypes (verified by DNA sequencing).

Data analysis. Because of the small number of subjects with some genotypes, we used CLUMP software with 10,000 Monte Carlo simulations to test for departures from Hardy-Weinberg equilibrium (15). We then conducted a bivariate and multivariate analysis of the association between APOE genotype and 24-mo MDI score. We first compared characteristics of subjects for whom there were all of the data of interest (umbilical cord blood lead levels, APOE genotype, MDI scores, and covariate data) with characteristics of subjects who were not included because of missing data. Univariate distributions of continuous variables were examined to determine departures from normality. In our bivariate analysis, we examined the distribution of demographics and lifestyle characteristics with respect to MDI scores. To adjust for potential confounding, we included maternal IQ, maternal years of education, infant sex, umbilical cord blood lead level, and gestational age as covariates in all regression models of APOE genotype and MDI scores. Finally, we explored the modifying effect of APOE genotype on the association between umbilical cord lead level and MDI score. Our underlying hypothesis was that the slope or shape of the association between umbilical cord lead level and MDI score among subjects with the APOE4 would differ according to the APOE allele status (E4 carrier versus E3 or E2). We stratified our data set into subjects with at least one copy of APOE4 and subjects who carried either APOE3 or APOE2. We repeated the multivariate regressions in each of these strata using umbilical cord lead levels as our exposure of interest adjusting for infant sex, maternal IQ, receiving calcium supplements, and gestational age. The $\beta$ coefficients and their $95 \%$ confidence intervals for the umbilical cord lead levels were then compared for each strata to determine whether there was evidence of effect modification. We also constructed smoothed plots of the relationship between umbilical cord lead levels and MDI scores stratified by APOE genotype to explore differences in the shape of the association among APOE4 carriers and noncarriers. To adjust for the covariates, the dependent variable of the smoothed plots was the residuals of the regression of MDI score by infant sex, maternal IQ, gestational age, and calcium supplementation. The independent variable was the residuals of the regression of umbilical cord lead by infant sex, maternal IQ, gestational age, and calcium supplementation. The regression was then stratified by APOE genotype and smoothed using a lowess smoothing function with a bandwidth of 0.8 .

\section{RESULTS}

Of the 424 infants with archived blood, 311 received a 24-mo BSID assessment. Umbilical cord blood specimens were obtained at delivery from $88 \%$ of newborns. Table 1 shows the basic demographic of the subjects included in the analysis and those excluded because of missing MDI score data. Subjects missing MDI data were similar with respect to the variables measured (Table 1). The results of the APOE genotyping (Table 2) were in Hardy-Weinberg equilibrium ( $p$ $=0.81$ ).

In the crude bivariate analysis, mean (SD) MDI scores at 24 mo of age were 94.2 (14.4) among infants with at least one copy of the APOE4 allele and $91.2(14.0)$ among the infants with either APOE2 or APOE3. This difference was not significant $(p=0.17)$. Mean umbilical cord blood lead levels did not differ between groups (APOE4 carriers: 7.0 (3.5); APOE2/E3 carriers $6.6(3.4) ; p=0.44)$.

Table 1. Demographics among subjects included in analysis and subjects with missing data

\begin{tabular}{lcc}
\hline \multicolumn{1}{c}{ Variable } & All data available $(n=311)$ & MDI missing $(n=113)$ \\
\hline Infant sex (\% male) & 54.3 & 58.4 \\
Maternal years of education Mean (SD) & $9.5(3.1)$ & $9.7(3.1)$ \\
Umbilical cord blood lead level $(\mu \mathrm{M} / \mathrm{L}$; mean $[\mathrm{SD}])$ & $0.32 \mu \mathrm{M} / \mathrm{L}(0.16)$ or $6.6(3.4) \mu \mathrm{g} / \mathrm{dL}$ & $0.31 \mu \mathrm{M} / \mathrm{L}(0.19)$ or $6.4(4.1) \mu \mathrm{g} / \mathrm{dL}$ \\
Maternal age at delivery & $24.9(5.4)$ & $24.5(4.2)$ \\
Gestational age $(\mathrm{wk})$ & $39.2(1.5)$ & $39.1(1.5)$ \\
Birth weight $(\mathrm{g})$ & $3155.8(415.1)$ & $3142.5(369.3)$ \\
Maternal IQ (mean SD) & $85.2(23.3)$ & $86.4(25.9)$ \\
MDI & $91.7(14.2)$ & - \\
APOE4 carrier prevalence $(N[\%])$ & $53(17 \%)$ & $21(19 \%)$ \\
\hline
\end{tabular}


Table 2. Results of genotyping among the 311 subjects with MDI scores and archived blood

\begin{tabular}{lccrc}
\hline & E2 & E3 & E4 & Totals \\
\hline E2 & 2 & 14 & 1 & 17 \\
E3 & - & 242 & 47 & 289 \\
E4 & - & - & 5 & 5 \\
Totals & 2 & 256 & 53 & 311 \\
\hline
\end{tabular}

Genotypes are read by combining the column and each row. For example, there are two subjects with the E2/E2 genotype, 14 who are E3/E2, etc. Allele frequency: E2, 19/622 (3\%); E3, 545/622 (88\%); E4, 58/622 (9\%). Allele carrier frequencies are as follows: $53(17 \%)$ subjects carry at least one copy of $\mathrm{E} 4,303(97 \%)$ carry at least one copy of E3, 17 (5\%) carry at least one copy of E2. Results are in Hardy-Weinberg Equilibrium $(p=0.81)$

Table 3 contains the results of the multivariate linear regression analysis. We should note that a significant positive effect of calcium supplementation was found; however, this is the focus of a separate study. There was no evidence of an interaction between calcium supplementation and APOE genotype (results not shown). After adjustment for potential confounding covariates, the effect of the APOE4 allele was clearer $(p<0.05)$. Because this effect may be due in part to effect modification of environmental exposures, we performed the same regression stratified by whether subjects carried the APOE4 allele or not (Table 4). We anticipated that our sample size (311 subjects total and 273 with cord blood, APOE genotype, and MDI score) would be underpowered to detect a statistically significant interaction between umbilical cord lead and APOE genotype. We did calculate the interaction term for APOE $4 \times$ umbilical blood lead $(\beta=0.6 ; 95 \%$ confidence interval: -0.7 to $1.8 ; p=0.4$ ). Nonetheless, there is an almost 3 -fold difference in the dose-response slopes between umbilical cord lead level and MDI score among APOE4 carriers and APOE2/APOE3 carriers. The direction of this effect suggests that subjects with APOE4 are relatively protected against the effects of developmental lead exposure. In the smoothed plots of MDI versus umbilical cord lead levels, subjects with E2 or E3 alleles have decreases in MDI scores at all levels of umbilical cord lead (Fig. 1). However, among carriers of the E4 allele, MDI scores only decreased with increasing blood lead levels among infants with the highest umbilical cord lead levels (Fig. 1). Such a finding might be expected if APOE4 is indeed protective against low-level lead toxicity.

\section{DISCUSSION}

We have demonstrated an association between APOE genotype and infant neurodevelopment. In this study, we found that the presence of the E4 variant was associated with an average
Table 4. Multiple linear regression analysis stratified by APOE genotype

\begin{tabular}{ccc}
\hline Variable & $\begin{array}{c}\text { Subjects without ApoE4 } \\
(N=218 ; \beta[95 \% \mathrm{CI}])\end{array}$ & $\begin{array}{c}\text { APOE4 carriers }(N= \\
44 ; \beta[95 \% \mathrm{CI}])\end{array}$ \\
\hline $\begin{array}{c}\text { Umbilical cord lead } \\
\text { level }(\mu \mathrm{g} / \mathrm{dL})\end{array}$ & $-0.77(-1.28$ to -0.25$)$ & $-0.18(-1.43$ to 1.08$)$ \\
\hline
\end{tabular}

APOE4 carriers defined as subjects with the E2/E4, E3/E4, or E4/E4 genotype. Subjects without APOE4 defined as subjects with E2/E2, E2/E3, or E3/E3 genotype. See Table 2 for carrier and allele frequencies.

4.4 point higher MDI score. Furthermore, among E4 carriers, a 1-unit change in umbilical cord lead level was associated with a 0.18-unit decrease in MDI score. Among E2/E3 carriers, the same change in blood lead was associated with a 0.77 -unit decrease in MDI score, suggesting possible protection against lead exposure among E4 carriers. ApoE is a cytoplasmic and serum transport protein for cholesterol and fatty acids that binds to LDL receptors and specific ApoE receptors $(16,17)$. ApoE is the main apolipoprotein secreted and produced within the brain parenchyma, where it is presumed to be involved in the redistribution of lipids among cells and in the regulation of cholesterol homeostasis, although other functions have been proposed, such as regulation of lipid peroxidation $(8,18,19)$. During the past $20 \mathrm{y}$, the requirement of the developing brain for essential fatty acids and cholesterol has received increasing attention (20). Although the focus has been particularly intent on long-chain polyunsaturated fatty acids and their role in brain growth and myelination, there is increasing evidence that cholesterol metabolism is also critical to brain development. For example, cholesterol binds to several synaptic proteins, is necessary for the formation of synaptic vesicles, and is critical for the clustering of postsynaptic receptors $(4,5)$. Defects in cholesterol metabolism are known to alter brain development. The Smith-Lemli-Opitz syndrome is attributed to a deficit in 7-dehydrocholesterol reductase and is characterized by holoprosencephaly, agenesis of the corpus callosum, and microcephaly (5). Furthermore, inhibitors of cholesterol synthesis in rats will also induce holoprosencephaly $(21,22)$.

In the 1990s, the association of the APOE4 allele with late-onset Alzheimer's disease spurred intense interest in the metabolism and neurologic function of this protein (23). Evidence suggests that ApoE plays an important role in neurite growth and may play an important role in processes that regulate synaptogenesis $(2,3,24)$. Our findings that ApoE isoforms predict developmental scores are consistent with previous studies suggesting that fatty acids and cholesterol metabolism are critical to brain development. Our results may

Table 3. Multivariate association of APOE4 genotype with MDI scores adjusting for covariates

\begin{tabular}{lccc}
\hline \multicolumn{1}{c}{ Variable } & \multicolumn{1}{c}{ } & $95 \%$ CI & $p$ \\
\hline APOE4* & 4.42 & 0.11 to 8.74 & \\
Umbilical cord lead level $(\mu \mathrm{g} / \mathrm{dL})$ & -0.68 & -1.15 to -0.21 & 0.04 \\
Male sex & -2.96 & -6.25 to 0.34 & 0.01 \\
Maternal IQ & 0.16 & 0.09 to 0.23 & $<0.01$ \\
Calcium supplementation & 3.60 & 0.35 to 6.9 & 0.03 \\
Gestational age (wk) & 1.07 & -0.06 to 2.20 & 0.06 \\
\hline
\end{tabular}

\footnotetext{
* APOE4 genotypes modeled as E4 carrier (one or two copies of E4 allele) vs E3 or E2 carriers (E3/E3, E3/E2, and E2/E2). See Table 2 for genotype
} frequencies. CI, confidence interval. 
even explain in part why the $\mathrm{E} 4$ variant is so common worldwide despite being associated with a terminal illness such as Alzheimer's disease. Presumably, beneficial effects of the E4 variant in early life would select for this variant to a greater degree than detrimental effects that manifest in the postreproductive years. This effect is sometimes referred to as antagonistic pleiotropy $(25,26)$.

The role of ApoE in mediating peripheral neuronal plasticity was first described in 1986 (27). After crushing of the sciatic nerve in the rat, local ApoE levels increased 100- to 200-fold relative to ApoE levels in controls. Furthermore, after nerve injury, ApoE levels correlated with nerve regeneration, peaking at $1 \mathrm{wk}$ postinjury and returning to baseline $8 \mathrm{wk}$ postinjury, at which time regeneration was complete (28). From these data, it has been proposed that ApoE scavenges cholesterol for neuronal regeneration (3). The regenerating nerve sends out growth cones or neurites expressing high levels of LDL receptors to recycle lipids and cholesterol, which are scavenged by ApoE for the purpose of new membrane synthesis. This model of nerve regeneration in the peripheral nervous system is well established and may serve as the model of ApoE function in the CNS as well $(17,28,29)$.

Perhaps of more relevance to neurodevelopment, the critical role of ApoE and cholesterol in synaptogenesis in the CNS was recently demonstrated. Glial cells are critical to synapse formation, but the signaling used is not clear. Using in vitro cell cultures, Mauch et al. (24) demonstrated that the synapse formation signal released by glial cells is cholesterol and that the primary signaling protein released by glial cells to promote cholesterol uptake and synaptogenesis is ApoE. APOE transcripts have been localized to astrocytes and microglia and are believed to be locally synthesized and secreted by glial cells and not by neurons $(5,30)$. Neuronal cells grown in culture will uptake ApoE, which has been suggested as evidence that ApoE regulates the intracellular neuronal transport of cholesterol and lipids (30). Similarly, neurons grown in culture without glial cells form only meager numbers of synapses with one another, further suggesting that the synapse-forming signal originates from glia $(24,30)$.

The critical role of ApoE in learning and memory has been demonstrated in behavioral as well as in vitro studies. APOE knockout mice provide informative research on effects of this gene on development and learning $(31,32)$. Behaviorally, ApoE-deficient mice display impairments in cognitive function in the Morris Water Maze test $(33,34)$. However, when these ApoE-deficient mice receive an injection of either E3 or E4 in the lateral ventricles, they demonstrate significant improvement in learning capacity in the Morris Water Maze test (35). This infusion also reversed the synaptodendritic pathology in these animals. The results of these studies further demonstrate that ApoE is critical to memory function more generally, not just in the aging brain.

Human data on ApoE and neurodevelopment are scant. Although ApoE isoforms have been studied extensively with respect to their role in Alzheimer's disease, the role of ApoE isoforms in childhood cognition and infant development has received little attention. Rask-Nissila et al. $(36,37)$ measured young children's intake of multiple dietary intake factors, including cholesterol and APOE phenotype, with respect to neuropsychiatric test scores. Overall differences in neuropsychiatric testing between E4 carriers and non-E4 carriers were not reported; however, failure rates on neuropsychiatric tests

\section{APOE2 or APOE3 Carrier}

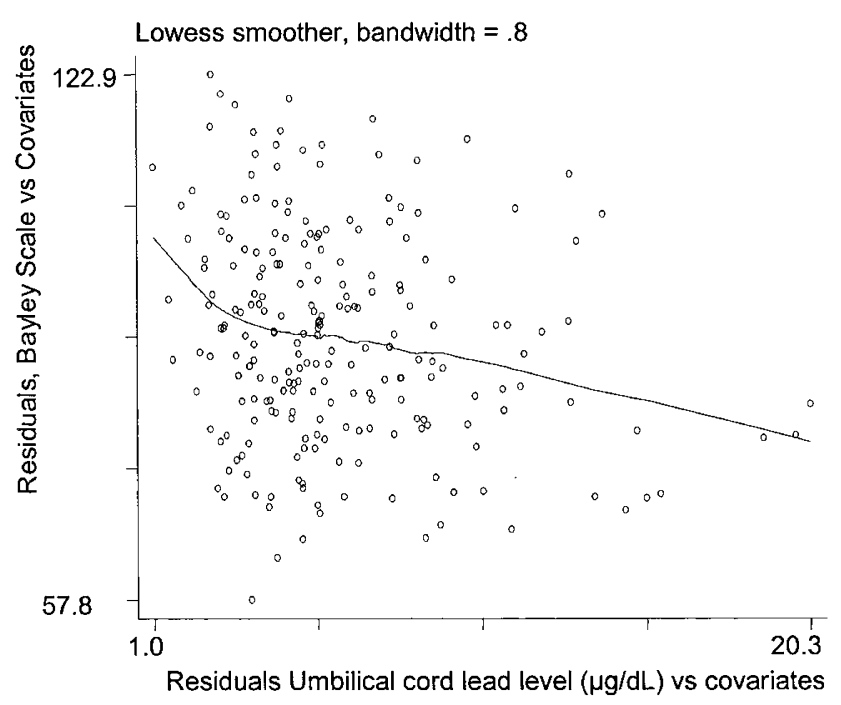

APOE4 Carrier

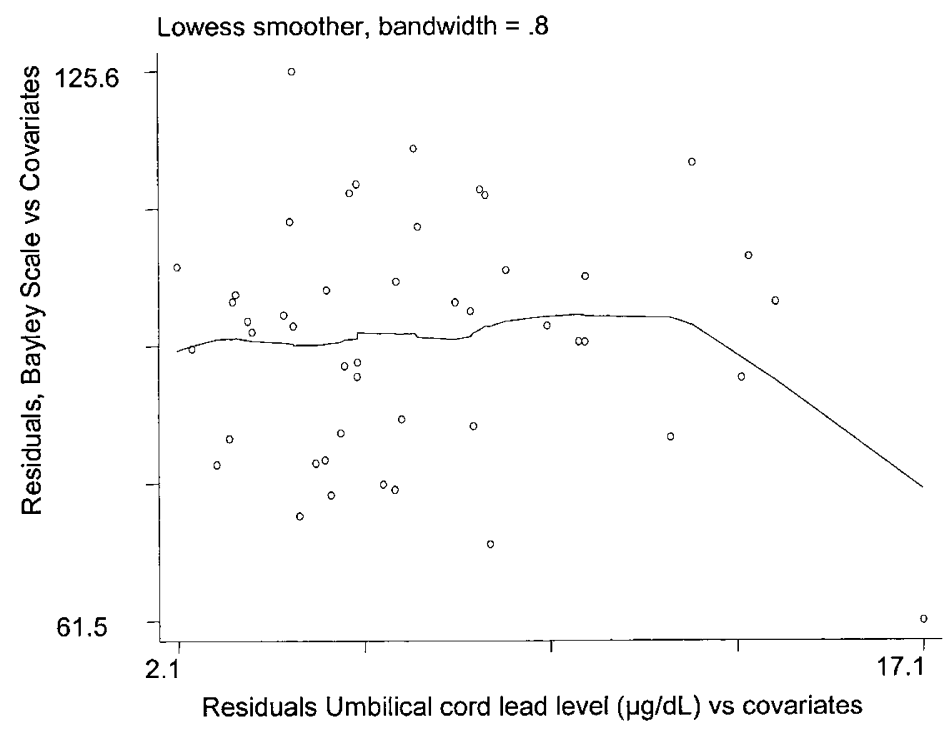

Figure 1. Smoothed plots of Bayley Mental Development Index score vs levels of lead in umbilical cord blood. Graphs represent the relationship among subjects with the E3 or E2 allele and among subjects with at 1 copy of the E4 allele. Graphs were constructed using the residuals of the Bayley Scale MDI score vs covariates (maternal IQ, infant sex, use of calcium supplements, and gestational age) for the Y axis, and the residuals of the regression of umbilical cord lead levels vs the same covariates for the $\mathrm{X}$ axis. For ease of interpretation we then "centered" these residuals by adding the overall mean Bayley scale score (91.7) to the $\mathrm{Y}$ variable and the overall mean umbilical cord blood lead level $(6.6 \mu \mathrm{g} / \mathrm{dL})$ to the $\mathrm{X}$ variable. By doing so, the graphs are adjusted for the covariates. 
performed at 5 y of age did not differ between groups (37). APOE4 carriers had lower failure rates on tests of speech and language skills ( 7.7 versus $10.4 \%$ ) although this difference was not significant. Likewise, Turic et al. (38) found no difference in a case-control study of 101 subjects with high general cognitive ability relative to 101 subjects with average cognitive ability when tested between 6 and 15 y of age. APOE 4 allele frequency in the high cognitive function group was $13.4 \%$ and in the average cognitive function group was $14.8 . \%(p=0.24)$. To our knowledge, ours is the first report of APOE genotypespecific performance on infant developmental tests. Our report of higher scores among E4 carriers is consistent with the study of Rask-Nissila et al. (37). Turic et al. (38) excluded poor performers from their study, which may have masked isoformspecific differences.

Neither of the two previous studies measured environmental toxins such as lead, another factor that may explain differences in our results. In our exploratory stratified analysis (Table 4), we found that the MDI score of subjects with E4 had a less steep dose response to umbilical cord lead levels than the response in subjects with E3 or E2, suggesting possible effect modification. Relative to the Rask-Nissila study, our analysis was in a poor urban population, where other unmeasured environmental toxins are more likely to be present. If APOE4 promotes myelination, synaptogenesis, or other fatty acid/ cholesterol-mediated processes associated with neurodevelopment, then the greater independent effect seen in our cohort relative to the Special Turku coronary risk factor intervention project for children (STRIP) study may be due protection against the adverse effects of neurotoxins on these processes. Further study is needed to verify these results and to determine whether exposure to environmental neurotoxins during early life is indeed modified by ApoE isoforms.

With respect to the limitations of this study, the predictive validity of scores on sensory-motor-based tests such as the BSID is limited. Prediction is stronger, however, the older the age at which the BSID is administered (i.e. the predictive validity is considerably greater for scores obtained at $2 \mathrm{y}$ of age than for scores obtained at 6 mo or $12 \mathrm{mo}$ of age). We also note that our sample size is small for testing effect modification, given that the APOE4 allele was present in only $17 \%$ of the study population. Our interaction analysis suggesting a protective effect of APOE4 on lead neurotoxicity therefore may be due to chance. In addition, we should note that early childhood blood lead levels predict 24-mo MDI scores independently of umbilical cord lead levels and may also interact with genetic variants in APOE to predict infant development. Our results should be considered exploratory and will need to be validated in further studies.

\section{CONCLUSION}

In summary, in a birth cohort of 311 infants, we found that the ApoE4 isoform predicted improved performance on the MDI score at 24 mo of age. Recent in vitro and gene knockout studies in animals support a biologic role for ApoE in neurodevelopment. The E4 isoform is associated with higher serum cholesterol, which is a necessary constituent for synaptogenesis (24). We believe that our results are consistent with evidence suggesting that ApoE and cholesterol regulate synapse formation and support the need for further research to determine the role of ApoE polymorphisms in predicting neurodevelopment.

Acknowledgments. The authors thank Dr. Lyle Palmer for assistance with the data analysis.

\section{REFERENCES}

1. Beffert U, Danik M, Krzywkowski P, Ramassamy C, Berrada F, Poirier J 1998 The neurobiology of apolipoproteins and their receptors in the CNS and Alzheimer's disease. Brain Res Brain Res Rev 27:119-142

2. Dietschy JM, Turley SD 2001 Cholesterol metabolism in the brain. Curr Opin Lipidol 12:105-112

3. Poirier J, Sevigny P 1998 Apolipoprotein E4, cholinergic integrity and the pharmacogenetics of Alzheimer's disease. J Neural Transm Suppl 53:199-207

4. Yankner BA 1996 Mechanisms of neuronal degeneration in Alzheimer's disease. Neuron 16:921-932

5. Teter B, Xu PT, Gilbert JR, Roses AD, Galasko D, Cole GM 1999 Human apolipoprotein $\mathrm{E}$ isoform-specific differences in neuronal sprouting in organotypic hippocampal culture. J Neurochem 73:2613-2616

6. Bothwell M, Giniger E 2000 Alzheimer's disease: neurodevelopment converges with neurodegeneration. Cell 102:271-273

7. Cooper JA, Howell BW 1999 Lipoprotein receptors: signaling functions in the brain? Cell 97:671-674

8. Herz J, Beffert U 2000 Apolipoprotein E receptors: linking brain development and Alzheimer's disease. Nat Rev Neurosci 1:51-58

9. Lanting CI, Boersma ER 1996 Lipids in infant nutrition and their impact on later development. Curr Opin Lipidol 7:43-47

10. Roux C, Dupuis R, Horvath C, Talbot JN 1998 Teratogenic effect of an inhibitor of cholesterol synthesis (AY 9944) in rats: correlation with maternal cholesterolemia. J Nutr 110:2310-2312

11. Wolf C, Chevy F, Pham J, Kolf-Clauw M, Citadelle D, Mulliez N, Roux C 1996 Changes in serum sterols of rats treated with 7-dehydrocholesterol-delta 7-reductase inhibitors: comparison to levels in humans with Smith-Lemli-Opitz syndrome. J Lipid Res 37:1325-1333

12. Weiss B 2000 Vulnerability of children and the developing brain to neurotoxic hazards. Environ Health Perspect 108(suppl 3):375-381

13. Lemasters GK, Perreault SD, Hales BF, Hatch M, Hirshfield AN, Hughes CL, Kimmel GL, Lamb JC, Pryor JL, Rubin C, Seed JG 2000 Workshop to identify critical windows of exposure for children's health: reproductive health in children and adolescents work group summary. Environ Health Perspect 108(suppl 3):505-509

14. Bellinger D, Leviton A, Waternaux C, Needleman H, Rabinowitz M 1987 Longitudinal analyses of prenatal and postnatal lead exposure and early cognitive development. N Engl J Med 316:1037-1043

15. Wasserman G, Graziano JH, Factor-Litvak P, Popovac D, Morina N, Musabegovic A Vrenezi N, Capuni-Paracka S, Lekic V, Preteni-Redjepi E 1992 Independent effects of lead exposure and iron deficiency anemia on developmental outcome at age 2 years. J Pediatr 121:695-703

16. Wigg NR, Vimpani GV, McMichael AJ, Baghurst PA, Robertson EF, Roberts RJ 1988 Port Pirie cohort study: childhood blood lead and neuropsychological development at age two years. J Epidemiol Community Health 42:213-219

17. Gomaa A, Hu H, Bellinger D, Schwartz J, Tsaih SW, Gonzalez-Cossio T, Schnaas L, Peterson K, Aro A, Hernandez-Avila M 2002 Maternal bone lead as an independent risk factor for fetal neurotoxicity: a prospective study. Pediatrics 110:110-118

18. Sham PC, Curtis D 1995 Monte Carlo tests for associations between disease and alleles at highly polymorphic loci. Ann Hum Genet 59:97-105

19. Mahley RW 1988 Apolipoprotein E Cholesterol transport protein with expanding role in cell biology. Science 240:622-630

20. Mahley RW, Rall SC Jr 2000 Apolipoprotein E: far more than a lipid transport protein. Annu Rev Genomics Hum Genet 1:507-537

21. Ramassamy C, Averill D, Beffert U, Bastianetto S, Theroux L, Lussier-Cacan S, Cohn JS, Christen Y, Davignon J, Quirion R, Poirier J 1999 Oxidative damage and protection by antioxidants in the frontal cortex of Alzheimer's disease is related to apolipoprotein E genotype. Free Radic Biol Med 27:544-553

22. Lovestone S 1999 Early diagnosis and the clinical genetics of Alzheimer's disease. J Neurol 246:69-72

23. Strittmatter WJ, Weisgraber KH, Huang DY, Dong LM, Salvesen GS, Pericak-Vance M, Schmechel D, Saunders AM, Goldgaber D, Roses AD 1993 Apolipoprotein E: high-avidity binding to beta-amyloid and increased frequency of type 4 allele in Alzheimer disease. Proc Natl Acad Sci U S A 90:1977-1981

24. Mauch DH, Nagler K, Schumacher S, Goritz C, Muller EC, Otto A, Pfrieger FW 2001 CNS synaptogenesis promoted by glia-derived cholesterol. Science 294:1354-1357

25. Caruso C, Candore G, Colonna Romano G, Lio D, Bonafe M, Valensin S, Franceschi C 2000 HLA, aging, and longevity: a critical reappraisal. Hum Immunol 61:942-949

26. Bailey JM 2000 How can psychological adaptations be heritable? Novartis Found Symp 233:171-180

27. Ignatius MJ, Gebicke-Harter PJ, Skene JH, Schilling JW, Weisgraber KH, Mahley RW, Shooter EM 1986 Expression of apolipoprotein E during nerve degeneration and regeneration. Proc Natl Acad Sci U S A 83:1125-1129 
28. Boyles JK, Zoellner CD, Anderson LJ, Kosik LM, Pitas RE, Weisgraber KH, Hui DY, Mahley RW, Gebicke-Haerter PJ, Ignatius MJ 1989 A role for apolipoprotein E, apolipoprotein A-I, and low density lipoprotein receptors in cholesterol transport during regeneration and remyelination of the rat sciatic nerve. J Clin Invest 83:1015-1031

29. Barres BA, Smith SJ 2001 Neurobiology. Cholesterol-making or breaking the synapse. Science 294:1296-1297

30. Ullian EM, Sapperstein SK, Christopherson KS, Barres BA 2001 Control of synapse number by glia. Science 291:657-661

31. Piedrahita JA, Zhang SH, Hagaman JR, Oliver PM, Maeda N 1992 Generation of mice carrying a mutant apolipoprotein $\mathrm{E}$ gene inactivated by gene targeting in embryonic stem cells. Proc Natl Acad Sci U S A 89:4471-4475

32. Gordon I, Genis I, Grauer E, Sehayek E, Michaelson DM 1996 Biochemical and cognitive studies of apolipoprotein-E-deficient mice. Mol Chem Neuropathol 28(13):97-103

33. Gordon I, Grauer E, Genis I, Sehayek E, Michaelson DM 1995 Memory deficits and cholinergic impairments in apolipoprotein E-deficient mice. Neurosci Lett 199:1-4
34. Oitzl MS, Mulder M, Lucassen PJ, Havekes LM, Grootendorst J, de Kloet ER 1997 Severe learning deficits in apolipoprotein E-knockout mice in a water maze task. Brain Res 752(1-2):189-196

35. Masliah E, Samuel W, Veinbergs I, Mallory M, Mante M, Saitoh T 1997 Neurodegeneration and cognitive impairment in apoE-deficient mice is ameliorated by infusion of recombinant apoE. Brain Res 751:307-314

36. Rask-Nissila L, Jokinen E, Terho P, Tammi A, Lapinleimu H, Ronnemaa T, Viikari J, Seppanen R, Korhonen T, Tuominen J, Valimaki I, Simell O 2000 Neurological development of 5-year-old children receiving a low-saturated fat, low-cholesterol diet since infancy: a randomized controlled trial. JAMA 284:993-1000

37. Rask-Nissila L, Jokinen E, Terho P, Tammi A, Hakanen M, Ronnemaa T, Viikari J, Seppanen R, Valimaki I, Helenius H, Simell O 2002 Effects of diet on the neurologic development of children at 5 years of age: the STRIP project. J Pediatr 140:328-333

38. Turic D, Fisher PJ, Plomin R, Owen MJ 2001 No association between apolipoprotein E polymorphisms and general cognitive ability in children. Neurosci Lett 299(12):97-100 